\title{
Entrées et sorties des médicaments hors GHS de la « liste en sus »
}

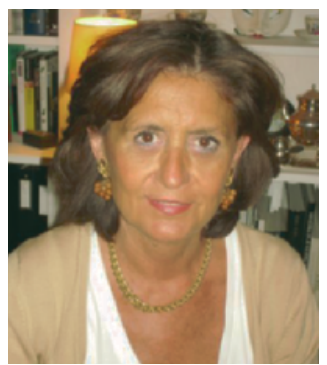

—M. Poivre

Pharmacien gérant,

Clinique Lambert, 92250 La Garenne Colombes

E-mail :martine.poivre@wanadoo.fr

$\mathrm{D}$ epuis la mise en place de la tarification à l'activité en 2005, certains médicaments, innovants et onéreux, sont pris en charge par l'Assurance maladie à condition que la prescription respecte AMM ou PTT et les différents Référentiels de bon usage des médicaments régulièrement mis à jour ; ce sont les médicaments hors GHS, inscrits sur la « liste en sus » et pour lesquels un tarif de responsabilité a été fixé par le Comité économique des produits de santé (CEPS).

Particulièrement dans le domaine de la cancérologie, cela a permis aux patients d'avoir accès aux traitements de chimiothérapie les plus innovants, dans tous les établissements, publics et privés ; les prescripteurs n'ont plus certaines contraintes budgétaires des années précédentes, mais le CBU contrôle la qualité de ces prescriptions.

- En mars 2005, la première liste de médicaments hors GHS est établie, le prix d'achat de tous les produits correspond au tarif de responsabilité fixé par le CEPS.

Il est défini que si l'établissement de santé achète à un prix inférieur au tarif de responsabilité il récupère la moitié du différentiel (EMI), l'autre moitié étant pour la Caisse d'Assurance maladie ; cela pour inciter à la négociation.

- Puis, certains médicaments de la « liste en sus » sont génériqués, ou ont des équivalents : Taxol ${ }^{\circledR}$, puis Farmorubicine ${ }^{\circledR}$, et Eloxatine ${ }^{\circledR}$, Navelbine ${ }^{\circledR}$, etc., les EPO ; leur prix d'achat baisse, générant des marges d'intéressement pouvant être très importantes en fonction des négociations. Cela permet d'aider à financer les demandes de plus en plus exigeantes des DRASS pour la mise en conformité des Unités de reconstitution, sous responsabilité pharmaceutique, le personnel, les soins, etc.

- Certains prix d'achat baissant fortement en 2009, les tarifs de responsabilité ont été revus à la baisse, entre autres pour ces médicaments, entraînant une diminution de la marge d'intéressement qui devenait quelquefois supérieure au prix d'achat!

- Le $1^{\text {er }}$ mars 2010, le paclitaxel, la vinorelbine, I'oxaliplatine, la fludarabine, l'épirubicine, médicaments très utilisés et qui sont des références dans le traitement des cancers du sein, de l'ovaire, du poumon et des cancers digestifs, sortent de la «liste en sus».

Cela engendre une baisse d'environ $20 \%$ du tarif versé aux établissements pour couvrir le coût de chimiothérapie. C'est un manque à gagner de l'EMI qui était très important pour certains de ces 5 médicaments ; mais, n'oublions pas qu'à la mise en place de la T2A on était remboursé à l'euro, l'euro ! II n'y avait pas ce différentiel qui a effectivement rapporté beaucoup d'argent, de plus en plus avec le temps, aux établissements de santé ! Ces derniers, surtout dans le privé, sont inquiets mais il n'y a pas possibilité de remplacer la prescription de ces produits par des produits restés hors GHS ou des formes orales qui posent le problème de l'observance... et ce ne serait pas nécessairement mieux pour le patient. Ces prescriptions qui rapportaient deviennent donc coûteuses, mais peu, le coût de la molécule étant maintenant très faible. Ces 5 molécules n'étant plus sur la « liste en sus", ils ne sont plus soumis aux Bonnes Pratiques des référentiels. N'est-il pas étonnant que les référentiels s'imposent seulement à certaines taxanes ?

- Plus de 5 génériques du docétaxel, de présentations diverses, sont prêts à être mis sur le marché dès la chute du brevet du Taxotère ${ }^{\circledR}$, prévue au plus tard au $1^{\text {er }}$ semestre 2011. Ils ont tous une formulation différente de celle du Taxotère ${ }^{\circledR}$.

La sortie de ces génériques entrainera la baisse du prix du Taxotère ${ }^{\circledR}$ et donc un EMI important. Mais cela ne remplacera pas les EMI perdus depuis le $1^{\text {er }}$ mars.

- Et courant 2011, la gemcitabine et l'irinotécan sortiront-ils de la « liste en sus » ou bien leur prix CEPS diminuera-t-il à nouveau?

À ce jour, en cancérologie, il n'y a plus que 5 molécules sur la « liste en sus » qui génèrent un EMI : la gemcitabine, l'irinotécan, Neorecormon ${ }^{\circledR}$, Eprex ${ }^{\circledR}$ et Aranesp ${ }^{\circledR}$, mais pour combien de temps? •

CEPS :

Comité économique des produits de santé

EMI :

écart moyen indemnisable

GHS :

groupe homogène de séjour 